\title{
Tangent Subdivision Scheme
}

\author{
Evelyne Vanraes \\ Adhemar Bultheel
}

Report TW 379, December 2003

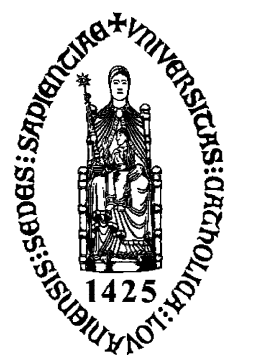

\section{Katholieke Universiteit Leuven} Department of Computer Science 


\title{
Tangent Subdivision Scheme
}

\author{
Evelyne Vanraes \\ Adhemar Bultheel
}

Report TW 379, December 2003

Department of Computer Science, K.U.Leuven

\begin{abstract}
In this paper we propose a new subdivision scheme based on uniform Powell-Sabin spline subdivision. For each vertex we have a control triangle tangent to the surface instead of a control point. The main advantage of this scheme is that we can choose the values of the tangent vectors in the initial vertices, which gives more design possibilities. Strictly speaking it is an approximating scheme, the control points for the vertices change each iteration. However, the point where the control triangle is tangent to the surface remains the same. Therefore in practice it is an interpolating scheme. In the regular regions we use the uniform Powell-Sabin rules and we develop subdivision rules for the new vertices in the neighbourhood of extraordinary vertices. The scheme yields $C^{1}$ continuous surfaces. We also do the convergence analysis based on the eigenproperties of the subdivision matrix and the properties of the characteristic map.
\end{abstract}

Keywords : Powell-Sabin splines, Generalized subdivision, Arbitrary topology, Subdivision matrix, Characteristic map.

AMS(MOS) Classification : 65D07, 65D17, 68U07. 


\title{
Tangent Subdivision Scheme
}

\author{
Evelyne Vanraes, Adhemar Bultheel
}

December 2003

\begin{abstract}
In this paper we propose a new subdivision scheme based on uniform Powell-Sabin spline subdivision. For each vertex we have a control triangle tangent to the surface instead of a control point. The main advantage of this scheme is that we can choose the values of the tangent vectors in the initial vertices, which gives more design possibilities. Strictly speaking it is an approximating scheme, the control points for the vertices change each iteration. However, the point where the control triangle is tangent to the surface remains the same. Therefore in practice it is an interpolating scheme. In the regular regions we use the uniform Powell-Sabin rules and we develop subdivision rules for the new vertices in the neighbourhood of extraordinary vertices. The scheme yields $C^{1}$ continuous surfaces. We also do the convergence analysis based on the eigenproperties of the subdivision matrix and the properties of the characteristic map.
\end{abstract}

Keywords: Powell-Sabin splines, Generalized subdivision, Arbitrary topology, Subdivision matrix, Characteristic map.

AMS(MOS) classification: 65D07,65D17, 68U07

\section{Introduction}

Subdivision schemes are defined procedurally as the limit of a refinement process on a coarse scale control net. When the subdivision process is iterated, the control net will contain more and more vertices. In the case of spline subdivision the control net converges to the spline surface described by the original control net. However, by the procedural definition, freedom from the closed mathematical expression can be achieved and one can define a subdivision process by an arbitrary linear map in the space of control points without knowing something a priori about the limit surface. If the sequence of control nets converges, one can use this procedure to generate a wide variety of surface types. These surfaces are no longer restricted to being images of bivariate functions, therefore they can easily represent shapes of arbitrary topology. Furthermore they support multiresolution algorithms such as level of detail rendering, multiresolution editing, compression and wavelets.

Subdivision schemes that generalize B-spline representations are particularly useful $[8,2]$. In the regular regions away from the extraordinary vertices the limit surface will be a spline surface and the basis functions are known. Spline based subdivision schemes are typically approximating. However, interpolating subdivision schemes for which the limit surface interpolates the initial control points are also of interest because the ability to control the resulting surface exactly is important in many applications. Modifications of approximating schemes have been proposed to force the limit surface to interpolate particular points and normals, but the resulting masks have infinite size [15] or the interpolation constraints are only satisfied in the limit $[9,6]$. Other schemes $[5,7]$ exist that are interpolating by design, but these are not spline based.

In this paper we present a new subdivision scheme that is spline based, but the limit surface interpolates a particular point and its normal for each initial vertex. It is a generalization of uniform 
Powell-Sabin spline subdivision [16]. For each vertex there is a control triangle instead of a control point, and this control triangle is tangent to the limit surface. The scheme is interpolating in the tangent point, tough the corners of the triangle vary with each iteration. The new control triangle lies in the same plane as the old control triangle, and therefor the scheme also interpolates the normals. We also give a convergence analysis based on the eigenanalysis of the subdivision matrix and the properties of the characteristic map [13, 10].

Section 2 gives a short overview of the idea behind subdivision schemes and reviews the main theorems for the convergence analysis. Section 3 introduces the tangent scheme and section 4 gives the convergence analysis of this new scheme. Finally section 5 gives some examples and discusses the drawbacks and advantages and gives suggestions for further research.

\section{Subdivision surfaces}

\subsection{The idea of subdivision}

A subdivision surface consist of two main ingredients: a coarse base polyhedron that controls the overall shape of the surface, and a refinement rule to compute inbetween control points on a finer scale. The surface is then defined as the limit of a sequence of successive refinements of the initial control polyhedron or polygonal mesh. Each control point on level $j+1$ can be computed as a linear combination of control points of the polyhedron on the previous level $j$. Figure 1 shows an example in which each triangle in the original mesh is split into four new triangles, quadrupling the number of triangles in the mesh.
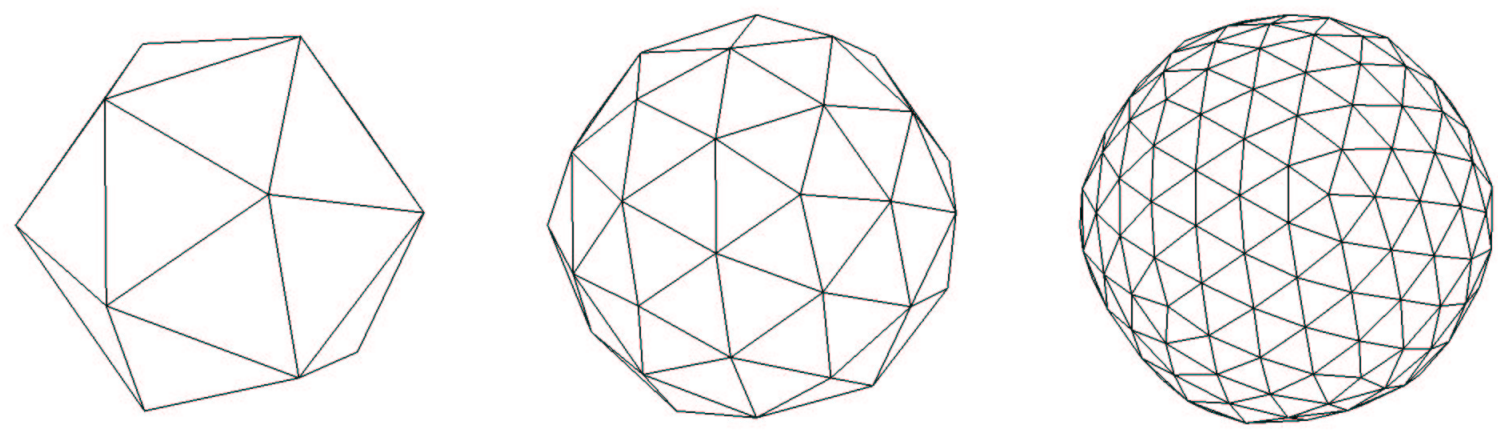

Figure 1. Example of a subdivision surface. The initial triangular mesh approximates the surface. Each triangle is split into 4, and the subdivision rule determines how the new vertices are computed.

Some subdivision methods generalize spline based knot insertion to irregular topology control meshes $[8,2]$, others are related to onedimensional interpolation schemes [5, 7]. The number of edges connected to a vertex may be different from vertex to vertex. As a result the rules derived from the spline basis functions or interpolation constraints can be applied only to those parts of the mesh that are locally regular; that is only to those vertices that have a valence of six for triangular schemes. For the vertices with other valences, called extraordinary vertices, additional rules are needed. This is the main challenge in the design of subdivision schemes for surfaces: one has to define additional 
rules for irregular parts of the mesh in such a way that the limit surfaces have desired properties, in particular, are smooth.

\subsection{The subdivision matrix and the characteristic map}

In general, a control net of three dimensional control points will consist of large regular regions, where standard continuity conditions can be used to describe a smooth surface, and isolated irregular regions, where these conditions are too restrictive. The key observation is that subdivision enlarges the regular regions of the control net and shrinks the irregular regions. The special rules used near the extraordinary vertices, do not change the number of extraordinary vertices in two consecutive meshes $\mathbf{p}^{j}$ and $\mathbf{p}^{j+1}$. The new vertices all have valence 6 . Since the subdivision masks have fixed finite size, we can restrict the analysis to nets $\mathbf{p}^{0}$ with a single extraordinary vertex surrounded by $r$ rings of ordinary vertices such that the regular parts of $\mathbf{p}^{0}$ define at least one complete surface ring.

If we denote by $\mathbf{s}^{j}$ the surface that corresponds to the regular parts of $\mathbf{p}^{j}$, then obviously $\mathbf{s}^{j-1}$ is part of $\mathbf{s}^{j}$ for $j \geq 1$, because each iteration the regular part is prolonged

$$
\mathbf{s}^{0} \subset \mathbf{s}^{1} \subset \mathbf{s}^{2} \subset \cdots
$$

and the limiting surface is given by $\mathbf{s}=\cup \mathbf{s}^{j}$. Taking $\mathbf{s}^{j-1}$ away from $\mathbf{s}^{j}$ we obtain a surface ring $\mathbf{r}^{j}$ which is added to $\mathbf{s}^{j-1}$ in the $j$ th iteration step. This yields

$$
\mathbf{s}=\mathbf{s}^{0} \cup \bigcup_{j \geq 1} \mathbf{r}^{j}
$$

At an extraordinary vertex of valence $n$ the surface $\operatorname{rings} \mathbf{r}^{j}$ can be parametrized over a common domain $\Omega \times \mathbb{Z}_{n}$ where $\Omega$ is

$$
\Omega=\{(u, v) \mid u, v \geq 0 \text { and } 1 \leq u+v \leq 2\} .
$$

The domain $\Omega \times \mathbb{Z}_{n}$ of the ring consist of $n$ copies of the unit $\Omega$ (figure 2 ).

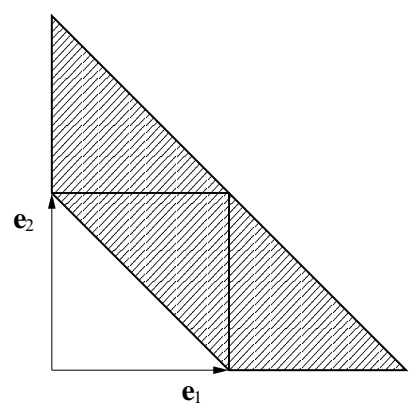

Figure 2: The unit domain $\Omega$

The control points that define the ring $\mathbf{r}^{j}$ are a subnet $\mathbf{d}^{j} \in \mathbf{p}^{j}$ and the corresponding regular basis functions that are used to parametrise the regular parts e.g. spline functions, are arranged in a row vector $\mathbf{B}$. The parametrisation is

$$
\mathbf{r}^{j}: \Omega \times \mathbb{Z}_{n} \rightarrow \mathbb{R}^{3}:(u, v, k) \rightarrow \mathbf{r}^{j}(u, v, k)=\sum_{i} \mathbf{d}_{i}^{j} B_{i}(u, v, k)=: \mathbf{B}(u, v, k) \mathbf{d}^{j} .
$$


The parts $\mathbf{r}_{k}^{j}(u, v)=\mathbf{r}^{j}(u, v, k)$ are referred to as segments and together they form the surface ring $\mathbf{r}^{j}$.

The subdivision algorithm can be described by a square subdivision matrix $S$ that relates the control points in the neighbourhood of a vertex on two sequential levels

$$
\mathbf{d}^{j}=S^{j} \mathbf{d}^{j+1} .
$$

A separate matrix is defined for each valence. The size of the subdivision matrix depends on the number of control points in $\mathbf{d}^{j}$ that is needed to define one surface ring. After one subdivision step the configuration of the involved vertices $\mathbf{d}^{j+1}$ is exactly similar to the configuration of the old vertices $\mathbf{d}^{j}$, and we can use the same numbering system. This is illustrated in figure 3 around an extraordinary vertex with valence 3 . In general the subdivision matrix can change from level to level. Most schemes are stationary which means that the same affine combinations are used in every step $j$ of the iteration and the subdivision matrix $S=S^{j}$ is independent of the level $j$.
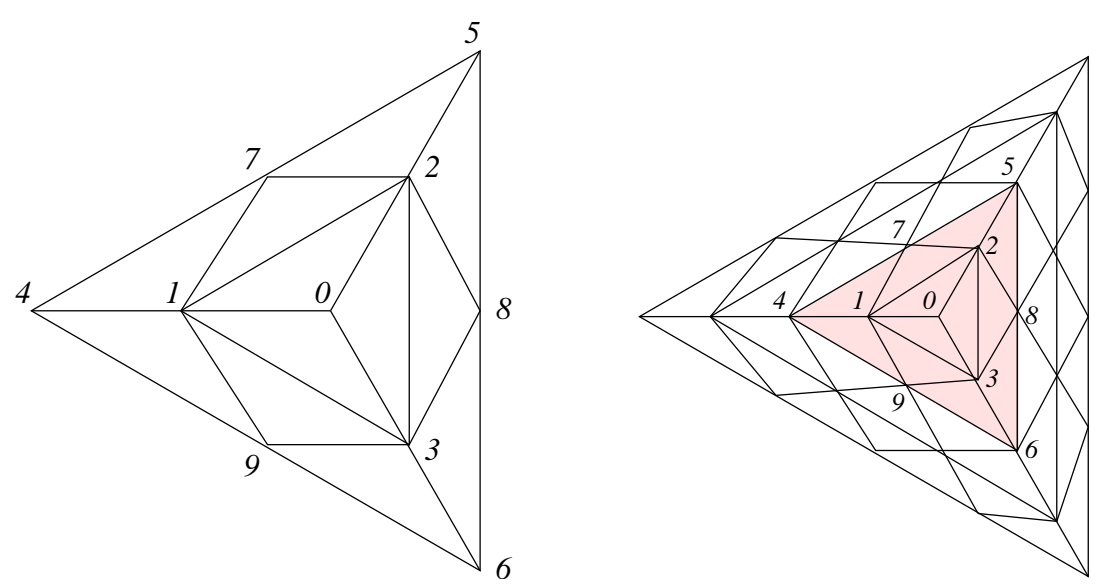

Figure 3: The vertices before and after subdivision have the same configuration.

Let $\lambda_{0}, \ldots, \lambda_{K}$ be the eigenvalues of $S$ ordered by their modulus

$$
\left|\lambda_{0}\right| \geq\left|\lambda_{1}\right| \geq \cdots \geq\left|\lambda_{K}\right|
$$

and denote by $\mathbf{v}_{0}, \ldots, \mathbf{v}_{k}$ the corresponding generalized eigenvectors. If $\left|\lambda_{0}\right| \geq\left|\lambda_{1}\right|=\left|\lambda_{2}\right|>\left|\lambda_{3}\right|$ the two dimensional surface that is defined by the twodimensional mesh $\left[\mathbf{v}_{1}, \mathbf{v}_{2}\right]$

$$
\Psi: \Omega \times \mathbb{Z}_{n} \rightarrow R^{2}:(u, v, k) \rightarrow \mathbf{B}(u, v, k)\left[\mathbf{v}_{1}, \mathbf{v}_{2}\right],
$$

or in complex form as

$$
\Psi^{*}: \Omega \times \mathbb{Z}_{n} \rightarrow C:(u, v, k) \rightarrow \mathbf{B}(u, v, k) \mathbf{v}^{*}, \quad \mathbf{v}^{*}:=\mathbf{v}_{\mathbf{1}}+i \mathbf{v}_{\mathbf{2}}
$$

is called the characteristic map of the subdivision scheme. Note that $\Psi$ can be regarded as consisting of $n$ segments $\Psi_{k}(u, v):=\Psi(u, v, k)$. In this paper we do not need the case where $\lambda_{1}$ is different from $\lambda_{2}$.

\subsection{Main theorem}

Most subdivision schemes are constructed from regular schemes, which are known to produce at least $C^{1}$ continuous surfaces for almost any initial configuration of control points. For the spline 
based systems, we know that the exact form of the limit surface in regular regions must be the spline surface itself, because it consist of pieces each influenced by a finite number of control points that are all regularly connected. The level of continuity is thereby well defined. For systems which are not spline based we cannot make this deduction straight away, but an analogous statement can be made after applying the same analysis as for the irregular points.

The main mathematical challenge in understanding the nature of subdivision surfaces is determining the behaviour around the extraordinary points. This was first analyzed for quadrilateral schemes by Doo and Sabin [4] using Fourier transforms and an eigenanalysis of the subdivision process. This work was expanded upon by Ball and Storry [1] who derived some necessary conditions. However, these are not sufficient because they only prove tangent plane continuity near the extraordinary point and self intersections are still possible. Reif [13] derived necessary and sufficient smoothness conditions by taking into account the eigenproperties of the subdivision matrix as well as the properties of the regular basis functions.

The crucial theorem for the analysis of subdivision algorithms can be stated in terms of the subdominant eigenvalue $\lambda:=\lambda_{1}=\lambda_{2}$ and the characteristic map $\Psi$. If $\lambda_{1}=\lambda_{2}, 1>|\lambda|>\left|\lambda_{3}\right|$ is a real eigenvalue with multiplicity 2 , and if the characteristic map $\Psi$ is regular and injective, then the limit surface is $C^{1}$ continuous for almost all initial control nets $\mathbf{d}^{0}$. A generalization with a less severe restriction on the eigenvalues can be found in [12].

In section 4 we will use this theorem to analyze the tangent scheme that we introduce in section 3. Checking if the characteristic map is regular and injective can be quite cumbersome and therefor we first discuss sufficient conditions for regularity and injectivity.

\subsection{Sufficient conditions for regularity and injectivity}

Assuming a double subdominant eigenvalue is less restrictive than it might seem at first since this case appears in a natural way for symmetric schemes. A subdivision scheme is said to be symmetric, if it is invariant under shifts and reflections of the labelling of the vertices in $\mathbf{d}_{m}$. This means if the permutation matrices $T$ and $R$ characterized by

$$
\begin{aligned}
\mathbf{B}(u, v, j+1) \mathbf{d}^{j} & =\mathbf{B}(u, v, j) T \mathbf{d}^{j} \\
\mathbf{B}(v, u,-j) \mathbf{d}^{j} & =\mathbf{B}(u, v, j) R \mathbf{d}^{j}
\end{aligned}
$$

exist, the subdivision matrix $S$ commutes with $T$ and $R$ :

$$
S T=T S \quad \text { and } \quad S R=R S .
$$

Symmetry implies that the subdivision matrix $S$ has a block-circulant structure with square blocks $S_{k}, k \in \mathbb{Z}_{n}$. Thus $S$ is unitary similar to a block-diagonal matrix $\hat{S}$. The diagonal blocks of $\hat{S}$ result from $S_{k}$ by the discrete Fourier transform

$$
\hat{S}_{k}=\sum_{l=0}^{n-1} \omega_{n}^{-k l} S_{l} \quad \text { for } k=0, \cdots, n-1,
$$

where $\omega_{n}=e^{\frac{2 \pi i}{n}}$ denotes an $n$-th root of unity. This means, if $\hat{\mathbf{v}}$ is an eigenvector of some block $\hat{S}_{k}$ corresponding to the eigenvalue $\mu$, then $\mu$ is also an eigenvalue of $S$ with complex eigenvector

$$
\mathbf{v}_{*}=\left[\omega_{n}^{0} \hat{\mathbf{v}}, \omega_{n}^{k} \hat{\mathbf{v}}, \cdots, \omega_{n}^{k(n-1)} \hat{\mathbf{v}}\right] .
$$

Because $S$ is real, the blocks of $\hat{S}$ satisfy $\hat{S}_{n-k}=\overline{\hat{S}}_{k}$ for $k=1, \cdots,\lfloor n / 2\rfloor$. Hence if $\mu$ is an eigenvalue of $\hat{S}_{k}$ it is also an eigenvalue of $\hat{S}_{n-k}$ and there are always two linear independent, real eigenvectors 
$\mathbf{v}_{1}=\operatorname{Re}\left(\mathbf{v}_{*}\right)$ and $\mathbf{v}_{2}=\operatorname{Im}\left(\mathbf{v}_{*}\right)$ corresponding to the real subdominant eigenvalue $\lambda$. It can be proven [10] that if the characteristic map is injective, the subdominant eigenvalue stems form the blocks $\hat{S}_{1}$ and $\hat{S}_{n-1}$. From now on we denote with $\hat{\mathbf{v}}$ the eigenvector of the subdominant eigenvalue corresponding to $\hat{S}_{1}$. A triangular subdivision scheme is said to have a normalised characteristic map, if $\hat{\mathbf{v}}$ is scaled such that $\Psi_{0}\left(\mathbf{e}_{1}+\mathbf{e}_{2}\right)=(p, 0)$ with $p>0$, where $\mathbf{e}_{1}$ and $\mathbf{e}_{2}$ are unit vectors in the $u$ and $v$ direction as in figure 2

For symmetric schemes we can restrict the analysis of the charateristic map to a single segment $\Psi_{0}$. Let $\Psi_{0}=\left[\Psi_{0}^{1}, \Psi_{0}^{2}\right]$ and denote by $\Psi_{0, v}:=\left[\Psi_{0, v}^{1}, \Psi_{0, v}^{2}\right]$ the partial derivative of $\Psi_{0}$ with respect to $v$. If the normalised characteristic map $\Psi$ of a symmetric subdivision scheme satisfies

$$
\Psi_{0, v}(u, v)>0 \quad \text { for all } \quad(u, v) \in \Omega
$$

componentwise, then the characteristic map is regular and injective. This was proven for quadrilateral schemes in [10] and extended to triangular schemes in [14].

\section{The tangent scheme}

The tangent scheme generalizes uniform Powell-Sabin spline subdivision to irregular settings.

\subsection{Powell-Sabin splines}

Powell-Sabin splines are piecewise quadratic polynomials with $C^{1}$ continuity on Powell-Sabin triangle splits. The PS-split $\Delta^{*}$ divides each triangle of the triangulation $\Delta$ into six smaller triangles with a common vertex as in figure 4 .

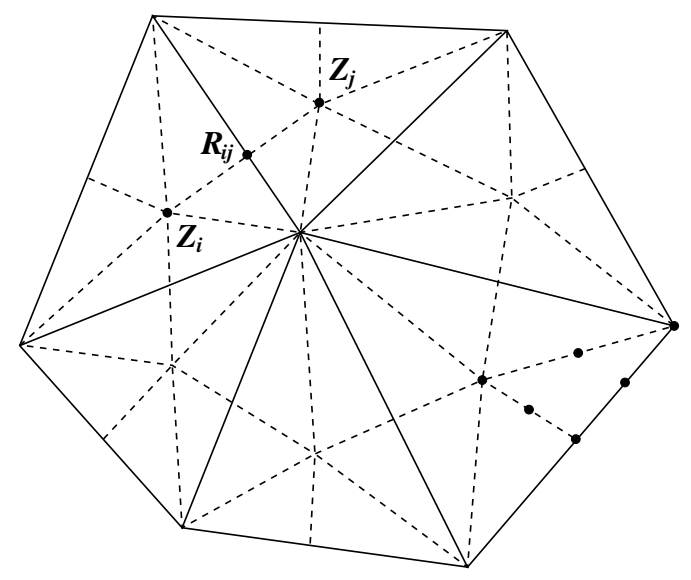

Figure 4. PS-refinement. Each triangle is split into six smaller triangles with a common vertex.

Each of the six small triangles is the domain of a quadratic Bernstein-Bézier polynomial. Powell and Sabin [11] proved that the dimension of the space $S_{2}^{1}\left(\Delta^{*}\right)$ equals $3 n$ : there exists a unique solution $s(x, y) \in S_{2}^{1}\left(\Delta^{*}\right)$ for the interpolation problem

$$
s\left(V_{k}\right)=f_{k}, \quad \frac{\partial s}{\partial x}\left(V_{k}\right)=f_{x, k}, \quad \frac{\partial s}{\partial y}\left(V_{k}\right)=f_{y, k}, \quad k=1, \ldots, n .
$$

So given the function and derivative values at each vertex $V_{k}$, the Bézier ordinates on the domain subtriangles are uniquely defined and the continuity conditions between subtriangles are automat- 
ically fulfilled.

\subsection{A normalised B-spline representation}

Dierckx [3] showed that each piecewise polynomial $s(x, y) \in S_{2}^{1}\left(\Delta^{*}\right)$ has a unique representation

$$
s(x, y)=\sum_{i=1}^{n} \sum_{j=1}^{3} c_{i j} B_{i}^{j}(x, y), \quad(x, y) \in \Omega,
$$

where the basis functions satisfy

$$
\begin{aligned}
& B_{i}^{j}(x, y) \geq 0 \\
& \sum_{i=1}^{n} \sum_{j=1}^{3} B_{i}^{j}(x, y) \equiv 1,
\end{aligned}
$$

and have local support: $B_{i}^{j}(x, y)$ is nonzero only on the so-called molecule $M_{i}$ of $V_{i}$, being the set of triangles $\rho_{l}$ that have $V_{i}$ as a vertex.

The construction of the basis functions yields for each vertex a triangle $t_{i}\left(Q_{i 1}, Q_{i 2}, Q_{i 3}\right)$ with vertices $Q_{i j}\left(X_{i j}, Y_{i j}\right)$ that must contain certain Bézier triangle points of the underlying Bézier representation. The triangles $t_{i}, i=1, \ldots, n$ are called PS-triangles.

We define the control points as

$$
C_{i j}=\left(Q_{i j}, c_{i j}\right)=\left(X_{i j}, Y_{i j}, c_{i j}\right)
$$

and the control triangles as

$$
T_{i}\left(C_{i 1}, C_{i 2}, C_{i 3}\right) .
$$

The projection of the control triangles $T_{i}$ in the $(x, y)$ plane are the PS-triangles $t_{i}$. One can prove that the control triangle $T_{i}$ is tangent to the surface $z=s(x, y)$ at $V_{i}$. The tangent point is $\left(x_{i}, y_{i}, s\left(V_{i}\right)\right)$.

\subsection{Uniform Subdivision}

For Powell-Sabin splines on uniform triangulation where all triangles are equilateral and have the same size, a subdivision scheme exists [16]. It is a dyadic scheme, a new vertex is inserted on every edge between two old vertices and every triangle is split into four new triangles.

For a new vertex inserted on the bottom edge as in figure 5 the new control triangle is found as follows

$$
\mathbf{p}_{c}^{j+1}=B \mathbf{p}_{a}^{j}+C \mathbf{p}_{b}^{j}
$$

with

$$
B=\left[\begin{array}{ccc}
0 & 1 / 2 & 1 / 2 \\
0 & 1 / 4 & 0 \\
0 & 0 & 1 / 4
\end{array}\right], \quad C=\left[\begin{array}{ccc}
0 & 0 & 0 \\
1 / 2 & 1 / 4 & 0 \\
1 / 2 & 0 & 1 / 4
\end{array}\right] .
$$

The control triangle for the old vertices needs to be rescaled

$$
\mathbf{p}_{a}^{j+1}=A \mathbf{p}_{a}^{j}
$$


with

$$
A=\left[\begin{array}{lll}
2 / 3 & 1 / 6 & 1 / 6 \\
1 / 6 & 2 / 3 & 1 / 6 \\
1 / 6 & 1 / 6 & 2 / 3
\end{array}\right]
$$

As mentioned before, the tangent point remains the same.
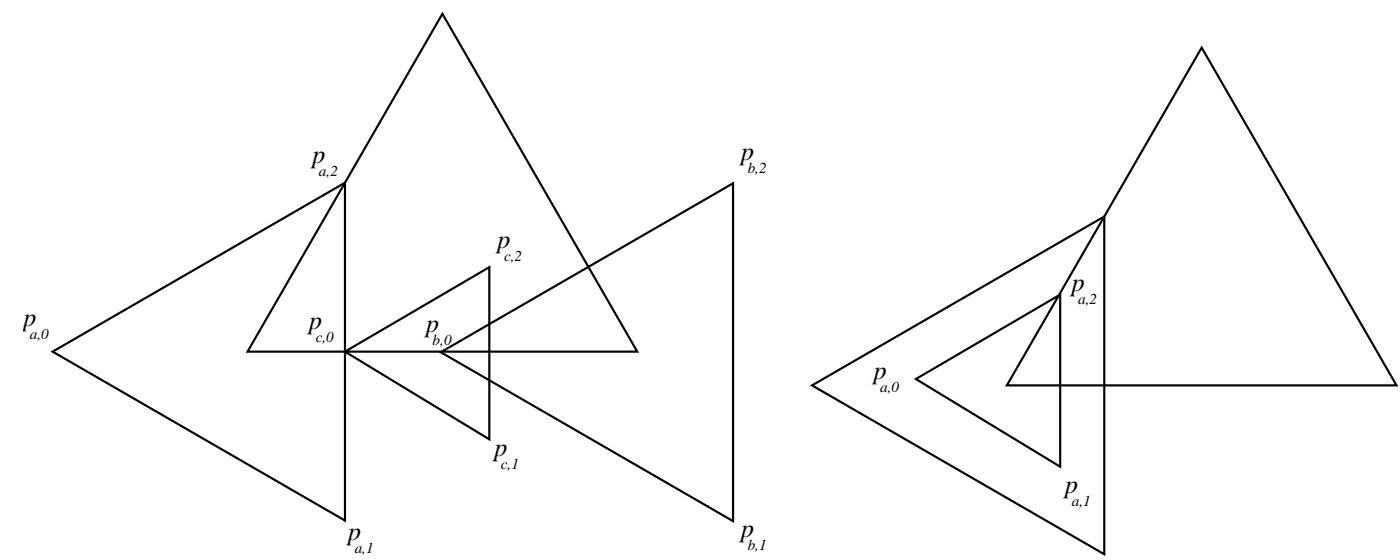

Figure 5: Subdivision of uniform Powell-Sabin splines.

For the derivation of these rules it was assumed that all control triangles point in the same direction and that their corners are numbered the same. However, in the neighbourhood of the extraordinary vertices where the different regular parts come together, it becomes impossible to request this. Therefore we also derive rules for other configurations of the control triangles. For example the new control triangle in figure 6 is found as

$$
\mathbf{p}_{e}^{j+1}=K \mathbf{p}_{b}+L \mathbf{p}_{d}
$$

with

$$
K=\left[\begin{array}{ccc}
0 & 0 & 0 \\
1 / 2 & 0 & 1 / 4 \\
1 / 4 & 0 & 1 / 2
\end{array}\right], \quad L=\left[\begin{array}{ccc}
1 / 2 & 1 / 2 & 0 \\
1 / 4 & 0 & 0 \\
0 & 1 / 4 & 0
\end{array}\right] .
$$

These rules can easily be derived from the underlying Bézier representation. The matrix $A$ for the rescaling of the old control triangle in equation (3.9) is independent of the orientation. Equations (3.7), (3.9) and (3.11) are matrix combinations because there are three control points for each vertex. Note that these equations are also valid if the domain triangles are not equilateral but only have all the same size as in figure 2 .

To simplify the analysis we reduce the number of rules that have to be derived by introducing the rotation matrix $Q_{6}$

$$
Q_{6}=\left[\begin{array}{ccc}
2 / 3 & 2 / 3 & -1 / 3 \\
-1 / 3 & 2 / 3 & 2 / 3 \\
2 / 3 & -1 / 3 & 2 / 3
\end{array}\right] .
$$

The effect of this matrix on a control triangle is a rotation of $2 \pi / 6=\pi / 3$ radians as illustrated in figure 7. We can now, after applying $Q_{6}$ an appropriate number of times, use equation (3.7) to compute a new vertex on every edge adjacent to the centroid. 


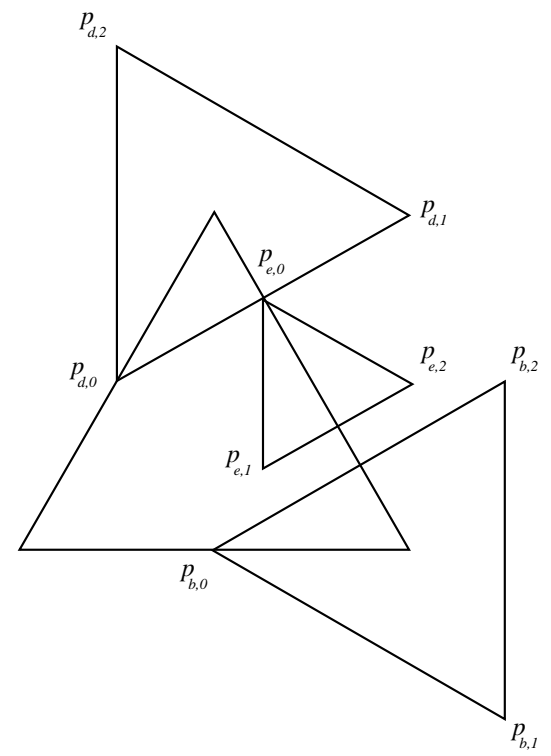

Figure 6: Rules for different orientations of the control triangles can be derived.
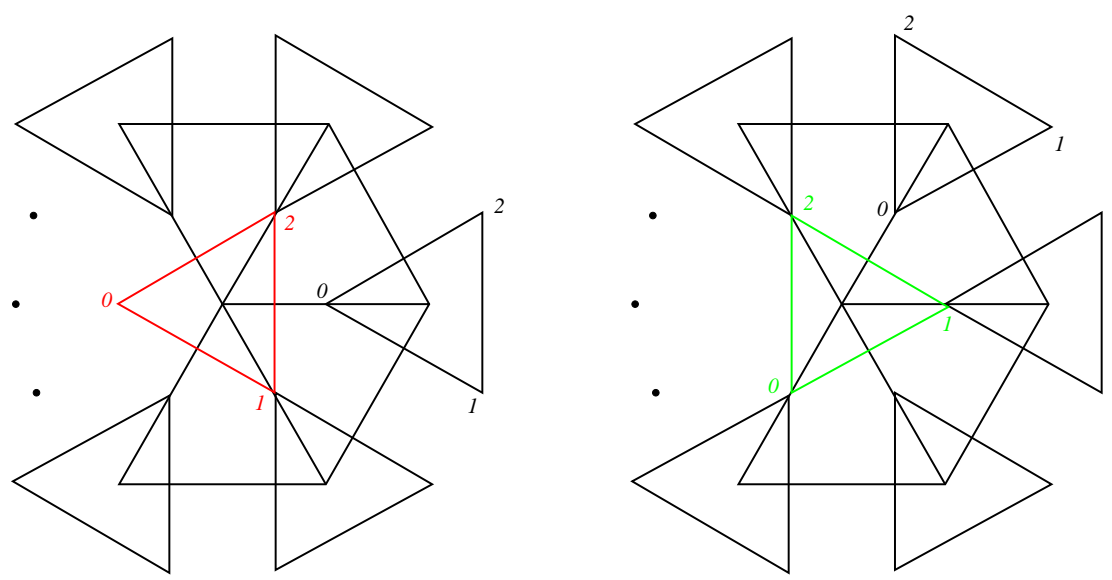

Figure 7: Rotation of the control triangle of the central vertex.

\subsection{Extraordinary vertices}

For the new rules in the extraordinary vertices we define a similar rotation matrix $Q_{n}$. It defines a rotation over an angle of $2 \pi / n$ radians

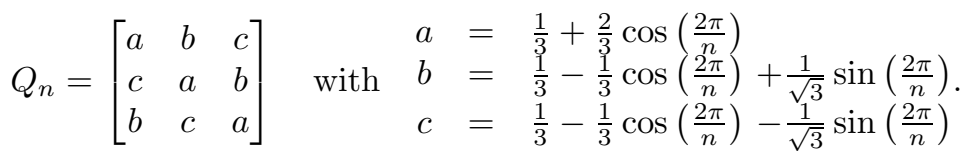

We now use exactly the same rules as in the uniform case, but use $Q_{n}$ instead of $Q_{6}$.

\section{Convergence Analysis}

To do the convergence analysis of the tangent scheme we first look at the structure of the subdivision matrix and calculate its eigenvalues. The subdominant eigenvalue turns out to be equal to $1 / 2$ and 
indeed stems form the blocks $\hat{S}_{1}$ and $\hat{S}_{n-1}$. For the analysis of the characteristic map we resort to the sufficient condition that the partial derivative of the first segment with respect to $v$ should be positive. We prove that this condition is fulfilled and we can conclude that the limit surface of the tangent scheme is $C^{1}$ continuous for almost all initial control nets $\mathbf{d}^{0}$.

\subsection{The subdivision matrix}

The form of the subdivision matrix depends on the labelling of the vertices in the control net $\mathbf{d}^{j}$. We label them segment after segment as in figure 8. For the tangent scheme only two rings around the vertex of interest are needed to do the analysis.

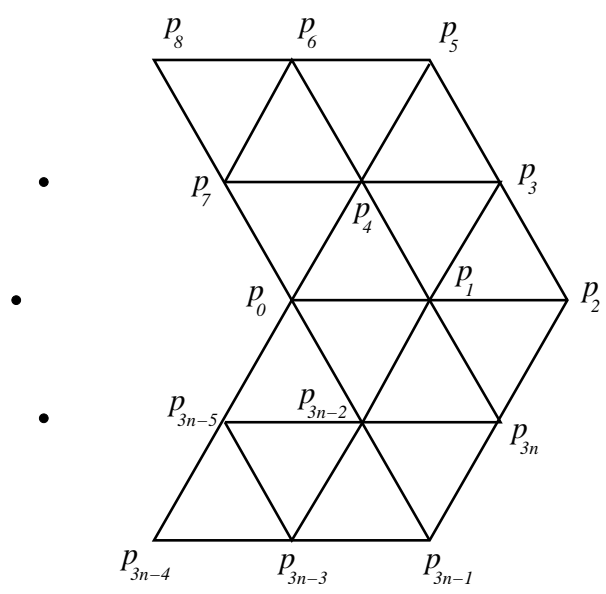

Figure 8: Labelling of the vertices

To achieve the desired periodic structure, we replace the centroid $\mathbf{d}^{0}$ by $n$ rotated copies

$$
\mathbf{d}_{0}^{j}=\frac{1}{n} \sum_{k=0}^{n-1} Q_{n}^{-j} \mathbf{d}_{0, k}^{j} .
$$

Tough not strictly necessary, we also replace the vertices $\mathbf{d}_{3 i+1}^{j}$ and $\mathbf{d}_{3 i+2}^{j}$ by two identical copies to facilitate the analysis of only a single segment of the characteristic map.

Then $S$ has a block circulant structure

$$
S=\left[\begin{array}{cccc}
S_{0} & S_{1} & \cdots & S_{n-1} \\
S_{n-1} & S_{0} & \cdots & S_{n-2} \\
\vdots & & \ddots & \vdots \\
S_{1} & \ldots & S_{n-1} & S_{0}
\end{array}\right]
$$

where

$$
S_{0}=\left[\begin{array}{cccccc}
\frac{1}{n} A & 0 & 0 & 0 & 0 & 0 \\
\frac{1}{n} B & \frac{1}{2} C & 0 & 0 & 0 & 0 \\
0 & \frac{1}{2} A & 0 & 0 & 0 & 0 \\
0 & \frac{1}{2} K & 0 & 0 & \frac{1}{2} L & 0 \\
\frac{1}{n} B Q_{n}^{-n+1} & 0 & 0 & 0 & \frac{1}{2} C & 0 \\
0 & 0 & 0 & 0 & \frac{1}{2} A & 0
\end{array}\right], \quad S_{1}=\left[\begin{array}{cccccc}
\frac{1}{n} A Q_{n}^{-1} & 0 & 0 & 0 & 0 & 0 \\
\frac{1}{n} B Q_{n}^{-1} & 0 & 0 & 0 & 0 & 0 \\
0 & 0 & 0 & 0 & 0 & 0 \\
0 & \frac{1}{2} L & 0 & 0 & 0 & 0 \\
\frac{1}{n} B & \frac{1}{2} C & 0 & 0 & 0 & 0 \\
0 & \frac{1}{2} A & 0 & 0 & 0 & 0
\end{array}\right]
$$


and

$$
S_{k}=\left[\begin{array}{cccccc}
\frac{1}{n} A Q_{n}^{-k} & 0 & 0 & 0 & 0 & 0 \\
\frac{1}{n} B Q_{n}^{-k} & 0 & 0 & 0 & 0 & 0 \\
0 & 0 & 0 & 0 & 0 & 0 \\
0 & 0 & 0 & 0 & 0 & 0 \\
\frac{1}{n} B Q_{n}^{-k+1} & 0 & 0 & 0 & 0 & 0 \\
0 & 0 & 0 & 0 & 0 & 0
\end{array}\right] \quad \text { for } \quad k=2, \cdots, n-1
$$

Now we can use the discrete Fourier transform to yield a unitary similar block-diagonal matrix $\hat{S}$ with blocks

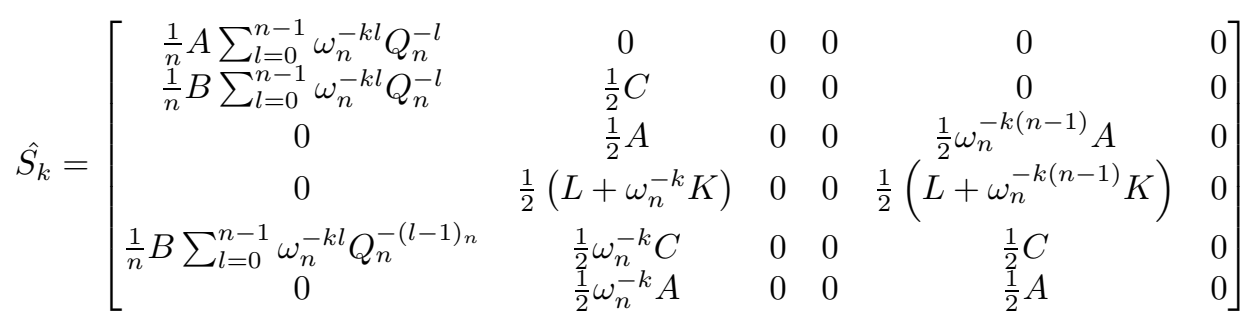

for $k \in \mathbb{Z}_{n}$. From this we get the eigenvalues of the subdivision matrix $S$ as in table 1 . The subdominant eigenvalue $\lambda$ equals $1 / 2$ and stems from $\hat{S}_{1}$ and $\hat{S}_{n-1}$.

\begin{tabular}{l|cc} 
& multiplicity & eigenvalue \\
\hline$\hat{S}_{0}$ & 1 & 1 \\
& 2 & $\frac{1}{4}$ \\
& 15 & 0 \\
\hline$\hat{S}_{1}$ and $\hat{S}_{n-1}$ & 1 & $\frac{1}{2}$ \\
& 2 & $\frac{1}{4}$ \\
$\hat{S}_{k}$ for $k \neq 0,1, n-1$ & 15 & 0 \\
& 2 & $\frac{1}{4}$ \\
& 16 & 0
\end{tabular}

ТАВ. 1. Eigenvalues of the blocks of the Fourier transformed subdivision matrix.

\subsection{The characteristic map}

What remains is the analysis of the characteristic map. Because of the convex hull property of the Powell-Sabin splines, the partial derivative of $\Psi_{0}$ with respect to $v$ will be positive, if all the Bézier coefficients in its Bézier representation are positive. 
The normalised eigenvector of $\hat{S}_{1}$ corresponding to the subdominant eigenvalue $\lambda=\frac{1}{2}$ is

$$
\hat{\mathbf{v}}=\left[\begin{array}{c}
-2+2 \sqrt{3} I \\
-2-2 \sqrt{3} I \\
4 \\
2-2 \sqrt{3} I \\
2-6 \sqrt{3} I \\
8-4 \sqrt{3} I \\
6-6 \sqrt{3} I \\
6-10 \sqrt{3} I \\
12-8 \sqrt{3} I \\
10+6 \sqrt{3} I \\
7-3 \sqrt{3} I \\
13-3 \sqrt{3} I \\
2+2 \sqrt{3} I \\
8+4 \sqrt{3} I \\
2+6 \sqrt{3} I \\
6+6 \sqrt{3} I \\
12+8 \sqrt{3} I \\
6+10 \sqrt{3} I
\end{array}\right] .
$$

The segment $\Psi_{0}^{*}$ of the characteristic map consist of 18 quadratic patches. The Bézier representation can be calculated from the B-spline coefficients (4.6) using the formulas from [3]. The result is given in table 2 .

$$
\begin{aligned}
& 8+8 I \\
& 7+7 I \quad 8+\frac{2}{3} I \quad 9+7 I \\
& 6+6 I \quad 7+\frac{17}{3} I \quad \frac{23}{3}+\frac{43}{9} I \quad \frac{17}{2}+\frac{29}{6} I \quad \frac{19}{2}+5 I \\
& 5+5 I \quad 6+\frac{14}{3} I \quad \frac{15}{2}+236 I \quad \begin{array}{ccc}
9 & 9+3 I & 10+3 I
\end{array} \\
& 4+4 I \quad 6+4 I \quad \frac{29}{4}+\frac{11}{4} I \quad \frac{17}{2}+\frac{3}{2} I \quad 10
\end{aligned}
$$

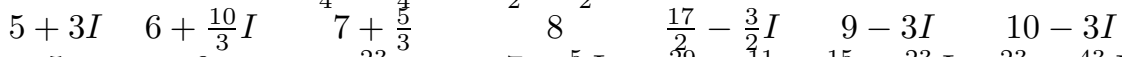

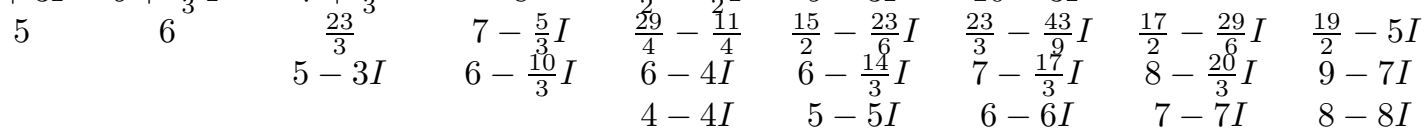

$$
\begin{aligned}
& 4-4 I \quad 5-5 I \quad 6-6 I \quad 7-7 I \quad 8-8 I
\end{aligned}
$$

TAB. 2. Bézier ordinates of $\Psi_{0}^{*}$.

The derivative of a Bézier polynomial in the direction $v$ is given as

$$
D_{v}^{1} b(\tau)=2 \sum_{i+j+k=1} b_{i j k}^{1}(v) B_{i j k}^{1}(\tau)
$$

where the coefficients $b_{i j k}^{1}$ are the intermediate results of the de Casteljau algorithm. Applying this to the Bézier ordinates in table 2 yields table 3.

Both the real and the imaginary part of the Bézier coefficients are positive and condition (2.13) is fulfilled. 


$$
\begin{array}{ccccc}
2+2 I & & & & \\
2+2 I & 2+2 I & \frac{5}{2}+\frac{5}{2} I & & \\
2+2 I & 2+2 I & 3+3 I & & \\
3+3 I & 2+\frac{10}{3} I & \frac{5}{2}+\frac{5}{2} I & 3+\frac{5}{3} I & \frac{7}{2}+\frac{3}{2} I \\
& & 4 & 4 & 4
\end{array}
$$

TAB. 3. Bézier ordinates of $\Psi_{0, v}^{*}$.

\section{Results}

\subsection{More design possibilities}

Figure 9 shows the results of the tangent scheme with a tetraeder as the initial control polygon. The configuration of the control triangles in the top row leads to a surface similar to the one one would get from an interpolating subdivision scheme like the Butterfly scheme [5]. Note that the control triangles are not shown in the figures, only the connected tangent points. The configuration of the bottom row starts with another steeper control triangle on the top of the tetraeder. The limit surface has a lob in the direction of the control triangle.
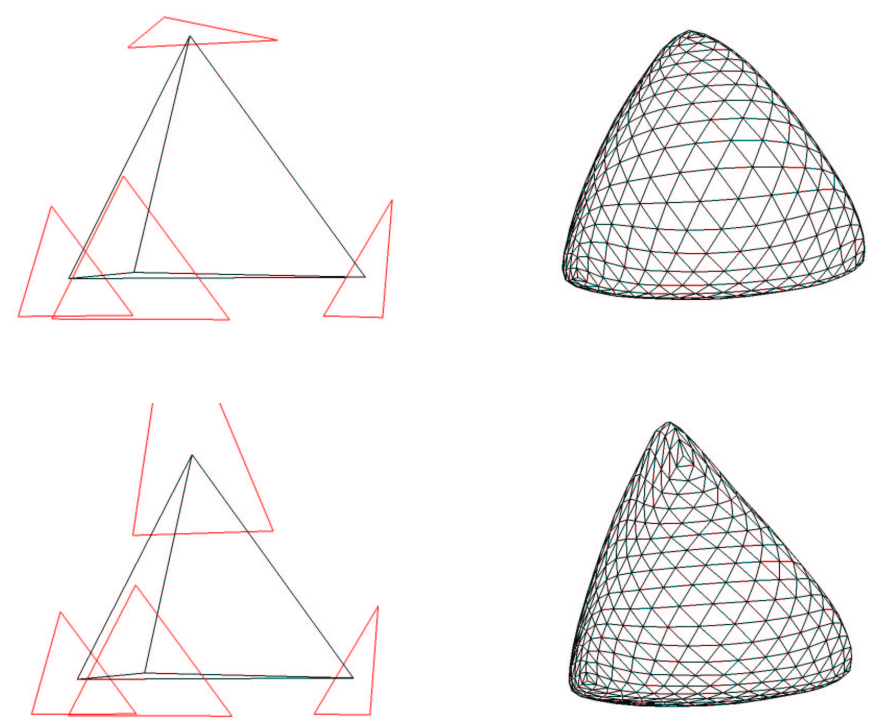

Figure 9: Choose the normal in initial vertices

Also the size of the control triangles influences the limit surface. Figure 10 shows the same tetraeder with different sizes for the control triangles. The smaller the control triangle, the sharper the corners. When the control triangles grows the corners become more rounded.

\subsection{Drawbacks}

Like most schemes the tangent scheme only yields $C^{1}$ continuous surfaces. To achieve $C^{2}$ continuity the schemes either have very large support, or have zero curvature at extraordinary vertices. Another problem is the presence of ripples in the neighbourhood of extraordinary vertices.

The tangent scheme is an expensive scheme as it comes to memory requirements. Instead of one 

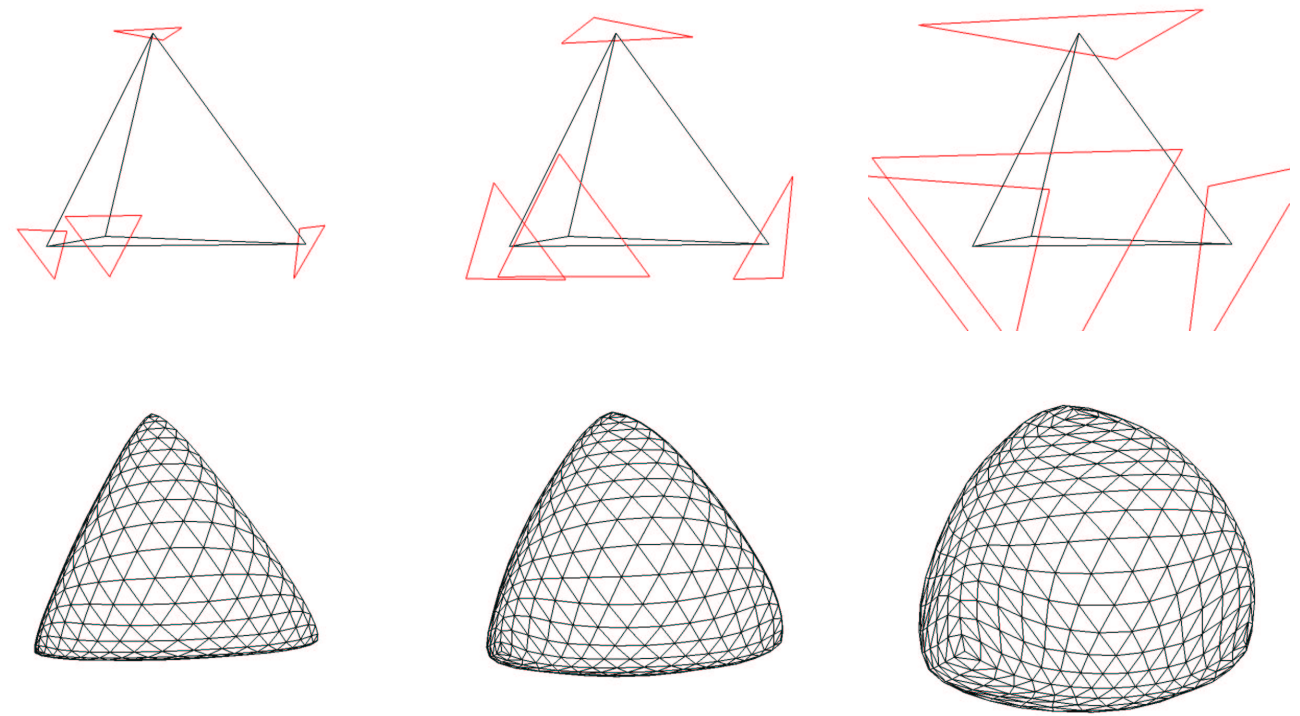

Figure 10: Effect of size of control triangles

control point we have three control points that need to be stored. Finally the question arises how the control triangles need to be determined in practical applications.

\subsection{Advantages}

Because the tangent scheme interpolates the initial tangent points and normals, we can control the limit surface more exactly. It also facilitates the design of subdivision wavelets or application as multiresolution editing.

The scheme is spline based. Therefore we have a closed mathematical expression for the surface in the regular regions and the limit positions are known.

The use of control triangles instead of control points gives us more design possibilities. We can choose the derivative in the initial tangent point and control the surface more exactly. The control triangles give us the tangents and normals for free which is useful for graphical display.

The stencil of the tangent scheme is compact, it uses only information of the immediate left and right neighbour of the new vertex and no information of vertices from neighbouring triangles. Therefor no special rules are needed for a surface with a boundary. Also cusps can be modeled by placing the control triangle perpendicular to the surface.

\section{Acknowledgement}

This work is partially supported by the Belgian Program on Interuniversity Poles of Attraction, initiated by the Belgian State, Prime Minister's Office for Science, Technology and Culture, and by the Flemish Fund for Scientific Research (FWO VLaanderen) project MISS (G.0211.02). The scientific responsibility rests with the authors. 


\section{Bibliography}

1. A. Ball and D. Storry. Conditions for tangent plane continuity over recursively generated B-spline surfaces. ACM Transactions on Graphics, 7(2):83-102, 1988.

2. E. Catmull and J. Clark. Recursively generated B-spline surfaces on arbitrary topological meshes. Computer Aided Design, 10(6):350-355, 1978.

3. P. Dierckx. On calculating normalized Powell-Sabin B-splines. Computer Aided Geometric Design, 15(3):61-78, 1997.

4. D. Doo and M. Sabin. Analysis of the behaviour of recursive division surfaces near extraordinary points. Computer Aided Design, 10(6):356-360, 1978.

5. N. Dyn, D. Levin, and J. Gregory. A butterfly subdivision scheme for surface interpolation with tension control. ACM Transactions on Graphics., 9(2):160-169, April 1990.

6. M. Halstead, M. Kass, and T. DeRose. Efficient, fair interpolation using catmull-calrk surfaces. In Computer Graphics Proceedings, Annual Conference Series, pages 35-44. ACM Siggraph, 1993.

7. L. Kobbelt. Interpolatory subdivision on open quadrilateral nets with arbitrary topology. In Proceedings of Eurographics 96, Computer Graphics Forum, pages 409-420, 1996.

8. C. Loop. Smooth subdivision surfaces based on triangles. Master's thesis, University of Utah, Departement of Mathematics, 1987.

9. A.H. Nasri. Surface interpolation on irregular networks with normal conditions. Computer Aided Geometric Design, 8:89-96, 1991.

10. J. Peters and U. Reif. Analysis of algorithms generalizing B-spline subdivision. SIAM Journal on Numerical Analysis, 35(2):728-748, April 1998.

11. M. J. D. Powell and M. A. Sabin. Piecewise quadratic approximations on triangles. ACM Transactions on Mathematical Software, 3:316-325, 1977.

12. U. Reif. Some new results on subdivision algorithms for meshes of arbitrary topology. In C.K. Chui and L.L Schumaker, editors, Wavelets and MultiLevel Approximation, Series in Approximations and Decompositions 2, pages 367-374. World Scientific, Singapore, 1995.

13. U. Reif. A unified approach to subdivision algorithms near extraordinary points. Computer Aided Geometric Design, 12:153-174, 1995.

14. G. Umlauf. Analyzing the characteristic map of triangular subdivision schemes. Constructive Approximation, 16(1):145-155, 2000.

15. A. Z. Averbuch V. A. Zheludev and M. Gruzd. Interpolatory subdivision schemes generated by splines. In A. Cohen, J.-L. Merrien, and L.L. Schumaker, editors, Curve and Surface Fitting: Saint-Malo 2002, pages 393-402. Nashboro Press, 2003.

16. J. Windmolders and P. Dierckx. Subdivision of Uniform Powell-Sabin splines. Computer Aided Geometric Design, 16:301-315, 1999. 